
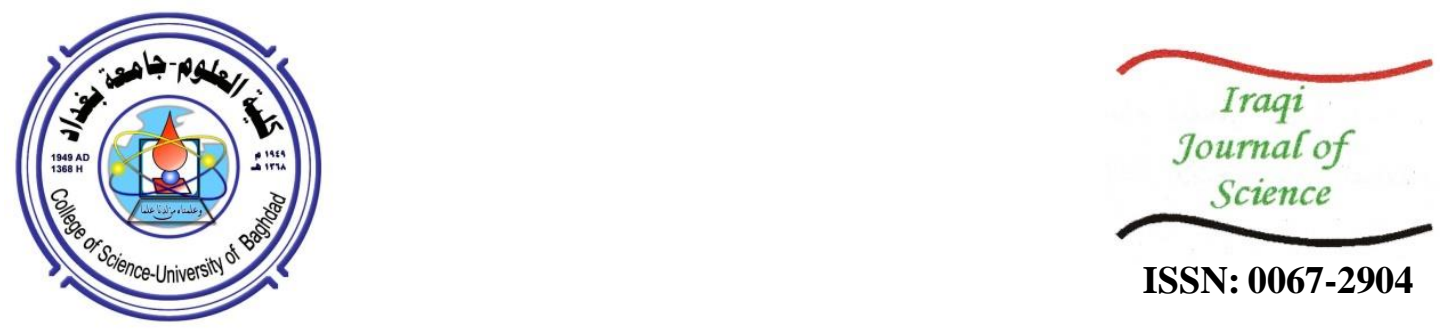

\title{
Soft Simply Compact Spaces
}

\author{
S. Noori*, Y. Y. Yousif \\ Department of Mathematics, College of Education for Pure Sciences, (Ibn- Al-Haitham), University of Baghdad \\ Received: 24/11/ 2019 \\ Accepted: 15/3/2020

\begin{abstract}
The aim of this research is to use the class of soft simply open set to define new types of separation axioms in soft topological spaces. We also introduce and study the concept of soft simply compactness.
\end{abstract}

Keywords: $S S^{M}$-compact, $S S^{M}-\mathrm{T}_{0}$ space, $S S^{M}-\mathrm{T}_{1}$ space.
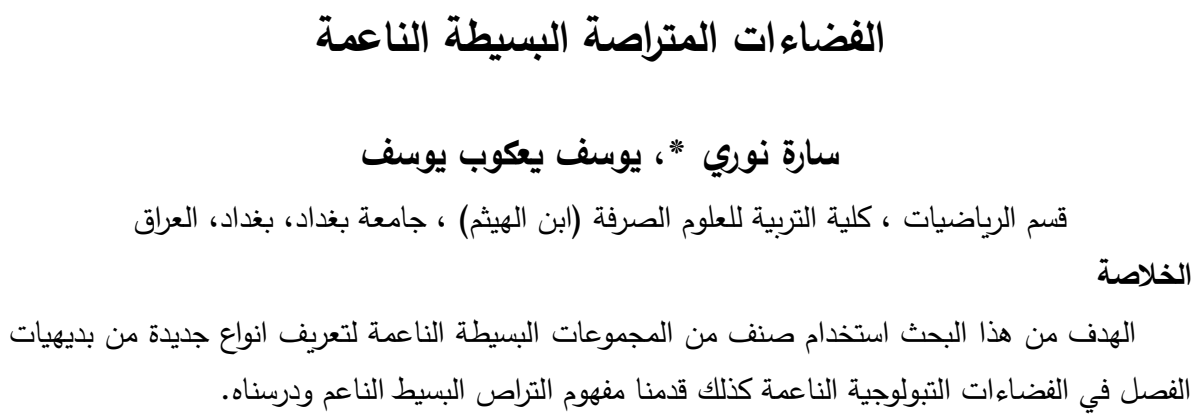

\section{Introduction}

Mathematics is established on exact notions where there is no ambiguity. In areas such as medicine, economy, engineering and sociology, the concepts are ambiguous and researchers want to predefine and study some new notions of ambiguity. They proposed several methods, such as the soft set theory, in order to overcome the uncertainty problem. In 1999, the concept of soft set theory was used for the first time as a mathematical tool by Molodtsov [1] to deal with confusion. He determined the primal upshots of this new theory and successfully applied the soft set theory in many ways, such as theory of measurement smoothness of functions, game theory, etc. In the last years, research on soft set theory was taking place rapidly. In 2003, Maji et al., presented many basic notions of soft set theory, including the universe soft set and empty soft set [2]. In 2011, Shabir and Naz discussed the theory of soft topological space and many fundamental concepts of soft topological spaces, including soft open, soft closed sets, soft nbd oft subspace, and soft separation axioms [3]. In 2012, Aygünoğlu and Aygün described the soft continuity of soft function, and they studied soft product topology, etc in soft topological spaces [4]. In 2011, Min discussed some findings on soft topological spaces [5]. In 1975, the concept of simply-open sets was introduced by Neubrunnova [6]; if ( $=K \cup N$ such that $K$ is open set and $N$ is nowhere dense ( $N$ is nowhere dense if $(\operatorname{cl}($ int $N)=\emptyset[7]))$, it symbolizes by $S^{M} O(X)$. In 2013 El. sayed and Noamman presented the transformed definition of simply open set [8]; if $(O \subset(X, \tau)$ is simply open set, then $\operatorname{int}(\operatorname{cl}(O)) \subseteq \operatorname{cl}(\operatorname{int}(O))$. In 2017, El.Sayed and El.Bably introduced a new class of simply open sets as a generalization and modification for soft open sets, which is called soft simply open set [9]. In this study, we build on some of the results from previous works [10-12]. The purpose of this paper is to introduce new concepts in soft topological spaces, such as $S S^{M}$-interior, $S S^{M}$-closure, $S S^{M}$-union, $S S^{M}$-intersection, $S S^{M}$-compact and soft simply separation axioms.

*Email: sarah.asas90@gmail.com 


\section{Preliminaries}

The following concepts and definition with some results are needed later on.

Definition 1.1: [1] Let $U$ be defined as a universe set and $E$ as a parameter set, with the power set of $U$ is denoted by $P(U)$ and $A c E$. Then $(F, A)$ is said to be a soft set, such that $F: A \rightarrow P(U)$; $F(a) \in P(U), \forall a \in A$.

Example 1.1: Let $U=\{2,3,4,7,9\}$ and $E=\left\{e_{1}, e_{2}, e_{3}\right\}$ where

$e_{1}=$ "even number ",$e_{2}=$ "odd number", $e_{3}=$ "prime number".

Suppose that $F\left(e_{1}\right)=\{2,4\}, F\left(e_{2}\right)=\{3,7,9\}, F\left(e_{3}\right)=\{2,3,7\}$, then

$(F, A)=\left\{\left(e_{1},\{2,4\}\right),\left(e_{2},\{3,7,9\}\right),\left(e_{3},\{2,3,7\}\right)\right\}$ is soft set.

Definition 1.2: [2]. We say that $(F, A)$ is a null set and it is symbolized by $\widetilde{\Phi}$, if $F(a)=\emptyset, \forall a \in A$.

Definition 1.3: [2]. We say that $(F, A)$ is an absolute soft set and it is symbolized by $\tilde{A}$, if $F(a)=$ $U, \forall a \in A$.

Definition 1.4: [2]. Let $(F, A)$ and $(G, B)$ are two soft sets, then $(F, A) \widetilde{U}(\mathrm{G}, \mathrm{B})=(H, C)$; (i.e the union of these sets is also a soft set), where $C=A \widetilde{\mathrm{U}} B$ and for each $e \in C$.

$\mathrm{H}(\mathrm{e})= \begin{cases}F(e) & \text { if } e \in A-B \\ G(e) & \text { if } e \in B-A \\ F(e) \cup G(e) & \text { if } e \in A \cap B\end{cases}$

Definition 1.5: [2]. Let $(F, A)$ and $(G, B)$ are two soft sets, then $(F, A) \widetilde{\cap}(\mathrm{G}, \mathrm{B})=(H, C)$; (i.e the intersection of these sets is also a soft set), where $C=A \widetilde{\cap} B$ and for each $e \in C$ such that $H(e)=$ $F(e) \cap G(e)$.

Definition 1.6: [2]. Let $(F, A)$ and $(\mathrm{G}, \mathrm{B})$ be two soft sets over $U$, then $(F, A) \widetilde{\subset}(\mathrm{G}, \mathrm{B})$, if $A \subset B$ and $F(e) \subset G(e) \forall e \in A$.

Definition 1.7: [13]. The soft topology $\tilde{\tau}$ is defined as follows:

1. $\widetilde{U}$ and $\widetilde{\emptyset} \in \tilde{\tau}$

2. The soft union of any number of soft sets in $\tilde{\tau} \in \tilde{\tau}$.

3. The soft intersection of any two soft sets in $\tilde{\tau} \in \tilde{\tau}$.

Then the triplet $(U, \tilde{\tau}, E)$ is said to be a soft topological space, the elements of $\tilde{\tau}$ are called soft open, their complements are soft closed, and we denote each of the closed soft sets by $\tilde{\mathcal{F}}$.

Definition 1.8: [13]. Assume that $(F, E)$ is a soft set of $(U, \tilde{\tau}, E)$ which is called soft neighborhood (briefly soft $n b d)$ subset $(H, E)$, if $\exists(K, E) \widetilde{\in} \tilde{\tau} ;(H, E) \widetilde{\subseteq}(K, E) \widetilde{\subseteq}(F, E)$.

Definition 1.9: [13]. $(F, E)^{o}$ or $\operatorname{sint}((F, E))$ is the soft interior of the set $(F, E)$ and is defined as follows:

$\operatorname{sint}((F, E))=\widetilde{U}\{(G, E) ;(F, E) \cong(G, E),(G, E) \widetilde{\in} \tilde{\tau}\}$.

Definition 1.10: [13]. $\overline{(F, E)}$ is a soft closure of a $(F, E)$ and is a soft set which is defined as follows: $\operatorname{scl}((F, E))=\widetilde{\cap}\left\{(G, E) ;(F, E) \widetilde{\subseteq}(G, E),(G, E)^{\complement} \widetilde{\in} \tilde{\tau}\right\}$.

Definition 1.11: [13]. We say that $(U, \tilde{\tau}, E)$ is a soft indiscrete space if $\tilde{\tau}=\{\widetilde{U}, \widetilde{\emptyset}\}$, and it is symbolized by $\tilde{\tau}_{\text {ind }}$.

Definition 1.12: [13]. We say that $(U, \tilde{\tau}, E)$ is a soft discrete space if $\tilde{\tau}$ is the family of all soft sets that can be defined over $U$ and is symbolized by $\tilde{\tau}_{\text {dis }}$.

Definition 1.13: [13]. We say that $(U, \tilde{\tau}, E)$ is a soft $T_{0}-$ space, if for any two distinct points $a, b \widetilde{\in} U$, there exist $(F, E)$ and $(G, E) \widetilde{\epsilon} \tilde{\tau}$, such that $[a \widetilde{\epsilon}(F, E)$ and $b \widetilde{\notin}(F, E)]$, or $[b \widetilde{\in}(G, E)$ and $a \widetilde{\notin}(G, E)]$.

Definition 1.14: [13]. We say that $(U, \tilde{\tau}, E)$ is a soft $T_{1}$-space, if for any two distinct points $a, b \widetilde{\in} U$, there exist $(F, E)$ and $(G, E) \widetilde{\epsilon} \tilde{\tau}$, such that $[a \widetilde{\in}(F, E), b \widetilde{\notin}(F, E)], \quad[b \widetilde{\in}(G, E)$ and a $\widetilde{\notin}(G, E)]$.

Definition 1.15: [13] We say that $(U, \tilde{\tau}, E)$ is a soft $T_{2}$ - space, if for any two distinct points $a, b \widetilde{\in} U$, there exist $(F, E)$ and $(G, E) \widetilde{\in} \tilde{\tau}$, such that $a \widetilde{\in}(F, E), b \widetilde{\in}(G, E)$ and $(F, E) \widetilde{\cap}(G, E)=$ $\widetilde{\emptyset}$.

Definition 1.16: [13] We say that $(U, \tilde{\tau}, E)$ is a soft regular space, if for all $(H, E) \widetilde{\in} \tilde{\tau}^{C}(i . e(H, E)$ is soft closed in $U$ ) and any points $a \widetilde{\in} U$ such that $a \widetilde{\notin}(H, E)$, then there exist $(F, E)$ and $(G, E) \widetilde{\epsilon} \tilde{\tau}$, such that $[a \widetilde{\epsilon}(F, E),(H, E) \widetilde{\subset}(G, E)$ and $(F, E) \widetilde{\cap}(G, E)=\widetilde{\emptyset}]$. 
Definition 1.17: [13] We say that $(U, \tilde{\tau}, E)$ is a soft normal space, if for each $(H, E),(K, E) \widetilde{\epsilon} \tilde{\tau}^{\mathrm{C}}$ (i.e $(H, E)$ and $(K, E)$ are soft closed in $U)$ such that $(H, E) \widetilde{\cap}(K, E)=\widetilde{\emptyset}$, then there exist $(F, E)$ and $(G, E) \widetilde{\in} \tilde{\tau}$, such that $[(H, E) \widetilde{\subset}(F, E),(K, E) \widetilde{\subset}(G, E)$ and $(F, E) \widetilde{\cap}(G, E)=\widetilde{\emptyset}]$.

Definition 1.18: [13]. We say that $(U, \tilde{\tau}, E)$ is a soft $T_{3}$ - space, if $U$ is both soft regular space and soft $T_{1}-$ space.

Definition 1.19: [13]. We say that $(U, \tilde{\tau}, E)$ is a soft $T_{4}-$ space, if $U$ is both soft normal space and soft $T_{1}-$ space.

Definition 1.20: [4]. A family $\delta$ of soft sets is called a cover of a soft set $(F, E)$ if $(F, E) \widetilde{\subset} \widetilde{U}\left\{\left(F_{i}, E\right) ;\left(F_{i}, E\right) \widetilde{\in} \delta ; i \in I\right\} . \delta$ is said to be a soft open cover if every members of $\delta$ is a soft open set.

Definition 1.21: [4]. We say that $(U, \tilde{\tau}, E)$ is a soft compact, if every soft open cover has a finite sub $\operatorname{cover}(U, \tilde{\tau}, E)$.

Definition 1.22: [8]. A soft subset $(F, A)$ of soft topological space $(U, \tilde{\tau}, E)$ is called Soft simply-open (for short $S S^{M}-$ open) set, if $\operatorname{sint}(\operatorname{scl}((F, A))) \widetilde{\subseteq} \operatorname{scl}(\operatorname{sint}((F, A)))$. It is symbolized by $S S^{M} O(U)$. The complement of a soft simply open set is a soft simply closed set (for short, $S S^{M}-$ closed) and it is symbolized by $S S^{M} C(U)$.

Remark 1.2: [8]. Every open soft set is a soft simply-open set but the converse is not true for the example:

Example 1.2: Let $U=\{a, b, c, d\}, \mathrm{E}=\left\{e_{1}, e_{2}\right\}$ and $\tilde{\tau}=\left\{\widetilde{U}, \widetilde{\varnothing},\left(F_{1}, E\right),\left(F_{2}, E\right),\left(F_{3}, E\right),\left(F_{4}, E\right)\right\}$ where:

$\left(F_{1}, E\right)=\left\{\left(e_{1},\{a, b\}\right),\left(e_{2},\{a, c, d\}\right)\right\}$

$\left(F_{2}, E\right)=\left\{\left(e_{1},\{a, b, d\}\right),\left(e_{2}, \widetilde{\varnothing}\right)\right\}$

$\left(F_{3}, E\right)=\left\{\left(e_{1},\{a, b\}\right),\left(e_{2}, \widetilde{\varnothing}\right)\right\}$

$\left(F_{4}, E\right)=\left\{\left(e_{1},\{a, b, d\}\right),\left(e_{2},\{a, c, d\}\right)\right\}$.

$\operatorname{Let}(G, E)=\left\{\left(e_{1},\{a, b\}\right),\left(e_{2},\{c, d\}\right)\right\}$, then

$\left(F_{4}, E\right)=\operatorname{sint}(\operatorname{scl}(G, E)) \widetilde{\subseteq} \operatorname{scl}(\operatorname{sint}(G, E))=U$

Thus $(G, E)$ is $S S^{M}$ - open set, but it is not open soft set .

Theorem 1.1: [8]. The following statement is introduced on $(U, \tilde{\tau}, E)$. Then

1. Let $\left(F_{1}, E\right)$ and $\left(F_{2}, E\right)$ are $S S^{M}$ - open sets, then $\left(F_{1}, E\right) \widetilde{U}\left(F_{2}, E\right)=\left(F_{3}, E\right)$, where $\left(F_{3}, E\right)$ is $S S^{M}$ - open set.

2. Let $\left(F_{1}, E\right),\left(F_{2}, E\right), \ldots,\left(F_{n}, E\right)$ are $S S^{M}-$ open sets, then $\left(F_{1}, E\right) \widetilde{\cap}\left(F_{2}, E\right) \widetilde{\cap} \ldots \widetilde{\cap}\left(F_{n}, E\right)$ is also $S S^{M}$ - open set.

Definition 1.23: [14]. We say that $(U, \tilde{\tau}, E)$ is a soft lindelö $f$, if every cover of $U$ has a countable sub cover.

\section{Main results}

In this section, we introduce the soft simply compact and define a new concepts in soft topological spaces like $S S^{M}$-interior, $S S^{M}$-closure, $S S^{M}$ - union, $S S^{M}$-intersection and soft simply separation axioms.

Definition 2.1: Let $(F, A)^{M}$ and $(G, B)^{M}$ be two soft simply sets then $(F, A)^{M} \widetilde{U}^{M}(G, B)^{M}=(H, C)^{M}$, where $C=A \cup B$ and for each $e \in C$

$$
\mathrm{H}(\mathrm{e})= \begin{cases}F(e) & \text { if } e \in A-B \\ G(e) & \text { if } e \in B-A \\ F(e) \cup G(e) & \text { if } e \in A \cap B\end{cases}
$$

Definition 2.2: Let $(F, A)^{M}$ and $(G, B)^{M}$ be two soft simply sets over $U$, then the soft simply intersection of them (for short $S S^{M}-$ intersection) is the soft simply set $(H, C)^{M}$, where $C=A \cap B$ and for all $e \in C$ such that $H(e)=F(e) \cap G(e)$ and denoted $(F, A)^{M} \widetilde{\cap}^{M}(G, B)^{M}=(H, C)^{M}$.

Definition 2.3: Let $(F, A)^{M}$ and $(G, B)^{M}$ be two soft simply sets over $U$, then $(F, A)^{M}$ is said to be soft simply subset of $(G, B)^{M}$ (for short $S S^{M}-$ subset) and denoted $(F, A)^{M} \widetilde{\subset}^{M}(G, B)^{M}$, if $A \subset B$ and $F(e) \subset G(e)$ for all $e \in A$.

Definition 2.4: $(F, A)^{C M}$ is a soft simply complement of $(F, A)^{M}$ (for short $S S^{M}$ - complement) which is defined as $F^{\mathrm{C}}(a)=U \backslash F(a), \forall a \in A$.

Definition 2.5: $S S^{M}\left(\operatorname{int}(F, E)^{M}\right)$ is a soft simply interior of a soft simply set $(F, E)^{M}$ (for short $S S^{M}$ - interior), which is a soft simply set defined as follows 
$S S^{M}\left(\operatorname{int}(F, E)^{M}\right)=\widetilde{\cup}^{M}\left\{(G, E)^{M} ;(G, A)^{M} \widetilde{\leftarrow}^{M}(F, E)^{M},(G, E)^{M} \in \tilde{\tau}\right\}$.

Definition 2.6: $S S^{M}\left(\operatorname{cl}(F, E)^{M}\right)$ is a soft simply closure of a soft simply set $(F, E)^{M}$ (for short $S S^{M}-$ closure $)$, which is a soft simply set defined as follows

$S S^{M}\left(\operatorname{cl}(F, E)^{M}\right)=\widetilde{\Omega}^{M}\left\{(G, E)^{M} ;(F, E)^{M} \widetilde{\subset}^{M}(G, E)^{M} ;(G, E)^{C^{M}} \in \tilde{\tau}\right\}$.

Definition 2.7: We say that $(U, \tilde{\tau}, E)$ is a soft simply $T_{0}-$ space (for short $S S^{M}-\mathrm{T}_{0}$ space) if for any two distinct points $a, b \in U$, then $\exists(F, E)^{M}$ and $(G, E)^{M} \in \tilde{\tau}$, such that $\left[a \in(F, E)^{M}, b \notin\right.$ $\left.(F, E)^{M}\right] \vee\left[b \in(G, E)^{M}\right.$ and $\left.a \notin(G, E)^{M}\right]$.

Definition 2.8: We say that $(U, \tilde{\tau}, E)$ is a soft simply $T_{1}-$ space (for short $S S^{M}-\mathrm{T}_{1}$ space) if for any two distinct points $a, b \in U$, then there exist $(F, E)^{M}$ and $(G, E)^{M} \in \tilde{\tau}$ such that $\left[a \in(F, E)^{M}\right.$, $\left.b \notin(F, E)^{M}\right],\left[b \in(G, E)^{M}\right.$ and $\left.a \notin(G, E)^{M}\right]$.

Definition 2.9: We say that $(U, \tilde{\tau}, E)$ is a soft simply $T_{2}-$ space (for short $S S^{M}-\mathrm{T}_{2}$ space) if for any two distinct points $a, b \in U$, then $\exists(F, E)^{M}$ and $(G, E)^{M} \in \tilde{\tau}$, such that $a \in(F, E)^{M}, \quad b \in$ $(G, E)^{M}$ and $(F, E)^{M} \widetilde{\cap}^{M}(G, E)^{M}=\emptyset$.

Definition 2.10: We say $(U, \tilde{\tau}, E)$ is a soft simply regular space (for short $S S^{M}$ - regular space) if for all $(H, E)^{M} \in \tilde{\tau}^{C}$ (i.e: $(H, E)^{M}$ is soft simply closed in $U$ ) and any points $a \in U$ such that $a \notin(H, E)^{M}$, then $\exists(F, E)^{M}$ and $(G, E)^{M} \in \tilde{\tau}$, such that $\left[a \in(F, E)^{M},(H, E)^{M} \widetilde{\subset}^{M}(G, E)^{M}\right.$, and $\left.(F, E)^{M} \widetilde{\cap}^{M}(G, E)^{M}=\emptyset\right]$.

Definition 2.11: We say that $(U, \tilde{\tau}, E)$ is a soft simply normal space (for short $S S^{M}$ - normal space) if for all $(H, E)^{M},(K, E)^{M} \in \tilde{\tau}^{C}$ (i.e: $(H, E)^{M}$ and $(K, E)^{M}$ are soft simply closed in $U)$ such that $(H, E)^{M} \widetilde{\cap}^{M}(K, E)^{M}=\emptyset$, then $\exists(F, E)^{M}$ and $(G, E)^{M} \in \tilde{\tau}$, such that $\left[(H, E)^{M} \widetilde{\subset}^{M}(F, E)^{M},(K, E)^{M} \widetilde{\subset}^{M}(G, E)^{M}\right.$ and $\left.(F, E)^{M} \widetilde{\cap}^{M}(G, E)^{M}=\varnothing\right]$.

Definition 2.12: We say that $(U, \tilde{\tau}, E)$ is a soft simply $T_{3}-$ space (for short $S S^{M}-\mathrm{T}_{3}$ space) if $U$ is both soft simply regular space and soft simply $T_{1}-$ space.

Definition 2.13: We say that $(U, \tilde{\tau}, E)$ is a soft simply $T_{4}-$ space (for short $S S^{M}-\mathrm{T}_{4}$ space) if $U$ is both soft simply normal space and soft simply $T_{1}-$ space.

$$
S S^{M}-\mathrm{T}_{4} \text { space } \rightarrow S S^{M}-\mathrm{T}_{3} \text { space } \rightarrow S S^{M}-\mathrm{T}_{2} \text { space } \rightarrow S S^{M}-\mathrm{T}_{1} \text { space }
$$

\section{Diagram 2.1}

Definition 2.14: A collection $\eta$ of soft simply sets is said to be soft simply cover of a soft simply set $(F, E)^{M}$, if $(F, E)^{M} \widetilde{\subset}^{M} U\left\{\left(F_{i}, E\right)^{M} ;\left(F_{i}, E\right)^{M} \in \eta ; i \in I\right\}$.

Definition2.15: A soft simply compact (for short $S S^{M}$ - compact), if every soft simply open cover that has a finite sub soft simply covers.

Definition 2.16: A soft simply lindelö $f$ (for short $S S^{M}$ - lindelöf), if each cover of $U$ by soft simply open sets has a countable sub cover.

Remark 2.1: Any $S S^{M}$ - compactt is $S S^{M}$ - lindelö $f$.

Proposition 2.1: A soft simply regular and $S S^{M}$ - lindelöf are $S S^{M}-$ normal.

Definition 2.17: If $(F, E)^{M}$ is a soft simply set of $(U, \tilde{\tau}, E)$, then we say that $(F, E)^{M}$ is a soft simply neighborhood (for short $S^{M}-n b d$ ) of the soft simply set $(H, E)^{M}$ if $\exists(K, E)^{M} \in \tilde{\tau}$ such that $(H, E)^{M} \subseteq(K, E)^{M} \subseteq(F, E)^{M}$.

Theorem 2.1: Any $S S^{M}$ - compact sub set of a $S S^{M}-\mathrm{T}_{2}$ space is $S S^{M}$ - closed.

Theorem 2.2: A soft simply closed subset of a soft simply compact space $(U, \tilde{\tau}, E)$ is a soft simply compact.

Proof: Let $(F, E)^{M} \in S S^{M} C(U)$, then $U \backslash(F, E)^{M} \in S S^{M} O(U)$. Suppose that $\left\{V_{i}: i \in I\right\}$ $\widetilde{\subset}^{M} S S^{M} O(U)$ be a cover of $(F, E)^{M}$, because $U$ is $S S^{M}$ - compact, then there exists a finite subsets $I_{0}$ of $I$ such that $U=U \backslash(F, E)^{M} \widetilde{U}^{M}\left(\widetilde{U}^{M}{ }_{i \in I_{0}} V_{i}\right) \in S S^{M} O(U)$. Hence $(F, E)^{M} \widetilde{\subset}^{M}\left(\widetilde{U}^{M}{ }_{i \in I_{0}} V_{i}\right)$ and $(F, E)^{M}$ are a $S S^{M}$ - compact sub set of $U$.

We obtain the following by combining theorems (2.1) and (2.2):

Corollary 2.1: A subset of a $S S^{M}-$ compact and $S S^{M}-\mathrm{T}_{2}$ space is $S S^{M}$ - compact if and only if it is $S S^{M}-$ closed.

Corollary 2.2: A $S S^{M}$ - compact and $S S^{M}-\mathrm{T}_{2}$ space is $S S^{M}-\mathrm{T}_{3}$ space .

Proof : Let $(F, E)^{M} \in U$, by Theorem $(2.1)(F, E)^{M}$, is $S S^{M}$ - closed, since there exists $\left(F_{1}, E\right)$, such that $\left(F_{1}, E\right)^{M}$ and $\left(F_{2}, E\right)^{M} \in S S^{M}-$ open, such that $e \in\left(F_{1}, E\right)^{M},(F, E)^{M} \widetilde{\subset}^{M}\left(F_{2}, E\right)^{M}$ and 
$\left(F_{1}, E\right)^{M} \widetilde{\cap}^{M}\left(F_{2}, E\right)^{M}=\varnothing$ (by proof from Theorem (2.1)). Hence, $U$ is $S S^{M}-$ regular. Since $U$ is $S S^{M}-\mathrm{T}_{2}$ space, by Diagram (2.1), then $U$ is $S S^{M}-\mathrm{T}_{1}$ space. Hence, $U$ is $S S^{M}-\mathrm{T}_{3}$ space.

Corollary 2.3: An $S S^{M}$ - compact and $S S^{M}-\mathrm{T}_{2}$ space are $S S^{M}-\mathrm{T}_{4}$ space.

Proof : Since $U$ is $S S^{M}$ - compact then $U$ is $S S^{M}$ - lindelöf (by Remark 2.1), and any $S S^{M}-$ compact and $S S^{M}-\mathrm{T}_{2}$ space are $S S^{M}-\mathrm{T}_{3}$ space (by Corollary 2.2), then $U$ is $S S^{M}-\mathrm{T}_{3}$ space. Hence, $U$ is $S S^{M}$ - regular space. Then $U$ is $S S^{M}$ - normal (by Proposition 2.1). Hence, $U$ is $S S^{M}-\mathrm{T}_{4}$ space.

Definition 2.18:[8] A function $f:(U, \tilde{\tau}, E) \rightarrow(V, \tilde{\sigma}, E)$ is said to be $S S^{M}$-irresolute such that $(U, \tilde{\tau}, E)$ and $(V, \tilde{\sigma}, E)$ are two soft topological spaces, if for each $(F, E)^{M} \in S S^{M} \mathrm{O}(\mathrm{V}), f^{-1}(F, E)^{M} \in S S^{M} O(U)$.

Theorem 2.3: Let $f:(U, \tilde{\tau}, E) \rightarrow(V, \tilde{\sigma}, E)$ is a $S S^{M}$-irresolute, where $(U, \tilde{\tau}, E)$ be a $S S^{M}-$ compact, then the image of a $S S^{M}$ - compact is $S S^{M}$ - compact.

Proof : Let $(F, E)^{M}$, by a $S S^{M}$ - compact , be a subset of $(U, \tilde{\tau}, E)$, and $\eta$ is the collection of $S S^{M}$-open sets cover of $\left(f(F, E)^{M}\right)$ in $(V, \tilde{\sigma}, E)$, such that $\left(f(F, E)^{M}\right) \widetilde{\subset}^{M} \widetilde{\cup}^{M}\left\{\left(F_{i}, E\right)^{M} ;\left(F_{i}, E\right)^{M} \in \eta ; i \in I\right\} . \quad$ Thus, $\left.(F, E)^{M} \widetilde{\subset}^{M} f^{-1}\left(f(F, E)^{M}\right)\right) \quad \widetilde{\subset}^{M} f^{-1}\left[\widetilde{U}^{M}\left\{\left(F_{i}, E\right)^{M} ; i \in I\right\}\right] \widetilde{\subset}^{M} \widetilde{\mathrm{U}}^{M}\left\{f^{-1}\left(F_{i}, E\right)^{M} ; i \in I\right\}$,

$(F, E)^{M} \widetilde{\subset}^{M} \widetilde{\mathrm{U}}^{M}\left\{f^{-1}\left(F_{i}, E\right)^{M} ; i \in I\right\}$. Hence, $f^{-1}(F, E)^{M}$ is $S S^{M}-$ open in $(U, \tilde{\tau}, E)$ (since $\mathrm{f}$ is $S S^{M}$ - irresolute function). Since $(F, E)^{M}$ is an $S S^{M}$ - compact and $\left\{f^{-1}\left(F_{i}, E\right)^{M} ; i \in I\right\}$ is an $S S^{M}$ - open cover of $(F, E)^{M}$, hence, $\exists$ is a finite subset $I_{0}$ of $I ;(F, E)^{M} \widetilde{U}^{M}\left\{f^{-1}\left(F_{i}, E\right)^{M} ; i \in\right.$ $\left.I_{0}\right\}$. Hence, $f(F, E){ }^{M} \widetilde{\complement}^{M} \widetilde{U}^{M}\left\{f f^{-1}\left(F_{i}, E\right)^{M} ; i \in I_{0}\right\} \widetilde{\subset}^{M} \widetilde{U}^{M}\left\{\left(F_{i}, E\right)^{M} ; i \in I_{0}\right\}$. Then $f(F, E)^{M}$ is $S S^{M}$ - compact.

Definition 2.19: Let $(U, \tilde{\tau}, E)$ and $(V, \tilde{\sigma}, E)$ be two soft topological spaces, then $(U, \tilde{\tau}, E)$ is said to be $S S^{M}$ - homeomorphic to $(V, \tilde{\sigma}, E)$ iff $\exists$ a $S S^{M}$-homeomorphism function ( $f$ is bij, $f$ is $S S^{M}$-irresolute, $f^{-1}$ is $S S^{M}$-irresolute) from $(U, \tilde{\tau}, E)$ onto $(V, \tilde{\sigma}, E)$, which we denoted as $(U, \tilde{\tau}, E) \cong{ }^{M}(V, \tilde{\sigma}, E)$.

Proposition 2.2: Let $f:(U, \tilde{\tau}, E) \rightarrow(V, \tilde{\sigma}, E)$ be an $S S^{M}$ - irresolute function from a compact topological space $(U, \tilde{\tau}, E)$ to a $S S^{M}-\mathrm{T}_{2} \operatorname{space}(V, \tilde{\sigma}, E)$. Then $f\left((F, E)^{M}\right)$ is closed in $(V, \tilde{\sigma}, E)$ for every $(F, E)^{M}$ is closed set $(U, \tilde{\tau}, E)$.

Theorem 2.4: The $S S^{M}$-irresolute and bijectione $f:(U, \tilde{\tau}, E) \rightarrow(V, \tilde{\sigma}, E)$ from a $S S^{M}-$ compact $(U, \tilde{\tau}, E)$ to $S S^{M}-\mathrm{T}_{2}$ space $(V, \tilde{\sigma}, E)$ are $S S^{M}$ - homeomorphism .

Proof: Let $\mathrm{g}:(V, \tilde{\sigma}, E) \rightarrow(U, \tilde{\tau}, E)$ be the $S S^{M}$ - inverse of the bijection $f:(U, \tilde{\tau}, E) \rightarrow(V, \tilde{\sigma}, E)$. If $(F, E)^{M}$ is $S S^{M}-$ open in $(U, \tilde{\tau}, E)$, then $(F, E)^{M^{C}}$ is $S S^{M}-$ closed in $(U, \tilde{\tau}, E)$, and hence $f(F, E)^{M^{C}}$ is $S S^{M}-$ closed in $(V, \tilde{\sigma}, E)$ (by Proposition 2.2). But $f(F, E)^{M^{C}}=g^{-1}\left((F, E)^{M^{C}}\right)=V \backslash$ $g^{-1}\left((F, E)^{M}\right)$. Hence, $g^{-1}\left((F, E)^{M}\right)$ is $S S^{M}-$ open in $(V, \tilde{\sigma}, E)$ for every $S S^{M}$-open set $(F, E)^{M}$ in $(U, \tilde{\tau}, E)$. Therefore, $\mathrm{g}:(V, \tilde{\sigma}, E) \rightarrow(U, \tilde{\tau}, E) \quad$ is $S S^{M}$-irresolute and thus $f$ is $S S^{M}$ - homeomorphism function.

Definition 2.20: A family $\delta$ of subsets of a soft topological space $(U, \tilde{\tau}, E)$ has a $S S^{M}$ - finite intersection property (for short $S S^{M}-f i p$ ) if each finite sub family of $\delta$ has non empty intersection .

Theorem 2.5 : A soft topological space $(U, \tilde{\tau}, E)$ is a $S S^{M}$ - compact if and only if any given collection of $S S^{M}$ - closed subsets of $U$ with the $S S^{M}-$ fip has non empty intersection.

Proof: Let $(U, \tilde{\tau}, E)$ be $S S^{M}$ - compact, and start with $\delta=\left\{\left(F_{i}, E_{i}\right)^{M} ; i \in I\right\}$ being a family of a $S S^{M}-$ closed sets of $U$ that has a $S S^{M}-f i p$. Then we want to prove that $\widetilde{n}_{i \in I}^{M}\left(F_{i}, E_{i}\right)^{M} \neq \emptyset$. Suppose that $\widetilde{\cap}_{i \in I}^{M}\left(F_{i}, E_{i}\right)^{M}=\emptyset$, then by the DeMorgan's law, $\left(\widetilde{\cap}_{i \in I}^{M}\left(F_{i}, E_{i}\right)^{M}\right)^{C}=\widetilde{\cup}_{i \in I}^{M}\left(F_{i}, E_{i}\right)^{M^{C}}=$ $U$. This implies that $\left\{\left(F_{i}, E_{i}\right)^{M^{C}} ; i \in I\right\}$ is a collection of $S S^{M}-$ open cover of , then $\exists I_{\alpha} \in I$ (soft simply sub set) such that $U=\widetilde{\cup}_{i \in I}^{M}\left(F_{i}, E_{i}\right)^{M^{C}}$ then $U^{C}=\left(\widetilde{\cup}_{i \in I}^{M}\left(F_{i}, E_{i}\right)^{M^{C}}\right)^{C}=\widetilde{\cap}_{i \in I_{\alpha}}^{M}\left(F_{i}, E_{i}\right)^{M}$. Therefore, $\widetilde{\cap}_{i \in I_{\alpha}}^{M}\left(F_{i}, E_{i}\right)^{M}=\emptyset$, which is a contradiction. Then $\widetilde{\cap}_{i \in I}^{M}\left(F_{i}, E_{i}\right)^{M} \neq \emptyset$.

Conversely, suppose that $\left\{\left(F_{i}, E_{i}\right)^{M} ; i \in I\right\}$ is a collection of an $S S^{M}$ - open cover of $U$. Let for all any finite subset $I_{\alpha} \in I$, we have $\widetilde{\cup}_{i \in I}^{M}\left(F_{i}, E_{i}\right)^{M} \neq U$, then $\left(\widetilde{\cap}_{i \in I_{\alpha}}^{M}\left(F_{i}, E_{i}\right)^{M}\right)^{C} \neq \emptyset$. Therefore, $\left\{\left(F_{i}, E_{i}\right)^{M^{C}} ; i \in I\right\}$ has an $S S^{M}-f i p$, we have $\left(\widetilde{\cap}_{i \in I_{\alpha}}^{M}\left(F_{i}, E_{i}\right)^{M}\right)^{C} \neq \emptyset$, which implies 
$\widetilde{\cap}_{i \in I_{\alpha}}^{M}\left(F_{i}, E_{i}\right)^{M} \neq U$, and this contradicts that $\left\{\left(F_{i}, E_{i}\right)^{M} ; i \in I\right\}$ is $S S^{M}-$ open cover of $U$, then $U$ is $S S^{M}-$ compact.

Definition 2.21: [8]. A function $f:(U, \tilde{\tau}, E) \rightarrow(V, \tilde{\sigma}, E)$ is said to be $S S^{M}-$ continuous such that $(U, \tilde{\tau}, E)$ and $(V, \tilde{\sigma}, E)$ are two soft topological spaces, if every $(F, E)^{M}$ is soft open set of $V$, then $f^{-1}(F, E)^{M}$ is $S S^{M}$ - open set of $U$.

Theorem 2.6 : Let $f:(U, \tilde{\tau}, E) \rightarrow(V, \tilde{\sigma}, E)$ between two soft topological spaces. Then $f$ is $S S^{M}-$ continuous if and only if the inverse image of each soft closed set in $(V, \tilde{\sigma}, E)$ is $S^{M}-$ closed in $(U, \tilde{\tau}, E)$.

Theorem 2.7: A soft continuous function is $S S^{M}$-continuous function.

Proof : Consider $f:(U, \tilde{\tau}, E) \rightarrow(V, \tilde{\sigma}, E)$ be a soft continuous function. Let $(G, E)$ be a soft open set in $V$, then $f^{-1}(G, E)$ is soft open in $U$, and so $f^{-1}(G, E)$ is $S S^{M}-$ open set in $U$. Therefore, $f$ is $S S^{M}$ - continuous function.

Theorem 2.8: Let $f:(U, \tilde{\tau}, E) \rightarrow(V, \tilde{\sigma}, E)$ and $g:(V, \tilde{\sigma}, E) \rightarrow(W, \tilde{\rho}, E)$ be two functions. Then $g \circ f:(U, \tilde{\tau}, E) \rightarrow(W, \tilde{\rho}, E)$ is $S S^{M}$ - continuous if $f$ is $S S^{M}$ - continuous and $g$ is soft continuous.

Proof : Consider $(H, E)^{M}$ be a soft closed set in $W$, because $g:(V, \tilde{\sigma}, E) \rightarrow(W, \tilde{\rho}, E)$ is soft continuous, then $g^{-1}(H, E)^{M}$ is soft closed set of $V$. Now $f:(U, \tilde{\tau}, E) \rightarrow(V, \tilde{\sigma}, E)$ is $S S^{M}$-continuous and $g^{-1}(H, E)^{M}$ is soft closed set of $V$, so (by Definition 2.25) $f^{-1}\left(g^{-1}(H, E)^{M}\right)=$ $(g \circ f)^{-1}(H, E)^{M}$ is $S S^{M}$-closed in $U$. Hence, $g \circ f:(U, \tilde{\tau}, E) \rightarrow(W, \tilde{\rho}, E)$ is $S S^{M}$-continuous.

\section{Conclusion}

In this paper, we introduced a new concept in soft topological spaces such as $S S^{M}$-interior, $S S^{M}$-closure, $S S^{M}$ - union, and $S S^{M}$-intersection. We also introduced the $S S^{M}$-compactness , studied some of its properties, and defined the soft simply separation axioms .

\section{References}

1. Molodtsov, D. 1999. Soft set theory-first results". Computers and Mathematics with Applications, 37(4-5): 19-31.

2. Maji P, K. and Biswas, R. and Roy, A. 2003. Soft set theory. Computers and Mathematics with Applications, 45(4-5): 555-562.

3. Shabir, M. and Naz, M. 2011. On Some New Operations in Soft Set Theory. Computers and Math.withAppl, 57: 1786-1799.

4. Aygünoğlu, A and Aygün, H. 2011. Some notes on soft topological spaces. Neural computing and Applications, 21(1): 113-119.

5. Min W. K. 2011. A note on soft topological spaces. Computers and Mathematics with Applications, 62(9): 3524-3528.

6. Neubrunnová, A. 1975. On transfinite sequences of certain types of functions. Acta Fac. Rer .Natur. Univ. Comenianae, 30: 121-126.

7. Willard, S. 1970. General topology. Addison Readings Mass. London D, on Mills. Ont.

8. El. Sayed, M and Noaman, I, A. 2013. simply fuzzy generalized open and closed sets . Journal of Advances in Mathematics, 4(3): 528-533.

9. El. Sayed, M and El-Bably, M. K. 2017. Soft Simply Open Sets in Soft Topological Space. Journal of Computational and Theoretical Nanoscience, 14(8): 4100-4103

10. Akdag, M and Ozkan, A. 2014. Soft b-open sets and soft b-continuous functions. Mathematical Sciences, 8(2): 124.

11. Benchalli, S, SPatil,P, G and Abeda, S, D. 2015. Soft $\beta$-Compactness in Soft Topological Spaces. Mathematical Sciences Internataional Research Journal, 4: 214-218.

12. Hussain, S and Ahmad, B. 2011. Some properties of soft topological spaces. Computers and Mathematics with Applications, 62(11): 4058-4067.

13. Shabir, M. and Naz, M. 2011. On soft topological spaces. Computers and Mathematics with Applications, 61(7): 1786-1799.

14. Rong, W. 2012. The countabilities of soft topological spaces. International Journal of Computational and Mathematical Sciences, 6: 159-162. 